\title{
Blood Flow Redistribution during Spontaneous Wheel Walk of the Rat
}

\author{
Yumi TAKEMOTo and Juro IRIUChIJIMA \\ Department of Physiology, Faculty of Medicine, University of Hiroshima, \\ Hiroshima, 734 Japan
}

\begin{abstract}
Changes in regional blood flow and arterial pressure in the rat during spontaneous walk in a wheel were observed. An electromagnetic flow probe was implanted around the carotid, superior mesenteric, or renal artery, or the terminal aorta and a catheter for pressure measurement was inserted into the terminal aorta or the common carotid artery. The wheel had a diameter of $35 \mathrm{~cm}$ and rotated passively as the rat walked. When hindquarter (terminal aortic) flow increased markedly during wheel walk, carotid flow decreased, superior mesenteric flow decreased or remained unchanged, and renal flow did not change. Arterial pressure remained almost unchanged and heart rate increased an average of about $10 \%$. Semiquantitative considerations indicated that arterial pressure was maintained in the face of the profuse increase in hindquarter flow during wheel walk by an increase in cardiac output rather than shifts of blood flow from other regions.
\end{abstract}

Key words: exercise, cardiac output redistribution, sympathetic vasoconstrictor fibers, heart rate, fixed action pattern.

Sudden increases in regional blood flow, without recruiting suitable compensatory mechanisms, might result in a sharp decrease in arterial pressure. In reality, arterial pressure is rarely decreased on such occasions, implying the presence of elaborate compensation. For the understanding of the compensatory mechanisms it is necessary to observe regional blood flow in animals under conditions which freely allow occasional increases in blood flow, i.e., in the conscious, unrestricted state. The laboratory animal rat is valuable for such studies because of the easiness of its handling and because it appears to live a contented life in relatively small artificially set spaces. Studies in rats are also expected to supplement the information accumulated in larger animals since FrankLIN et al. (1964).

Recent improvement of the electromagnetic flowmeter has made it possible to record blood flow in arteries in such small animals as the rat. By this method we

Received for publication February 13, 1989 
have observed that superior mesenteric and renal flows decrease when hindquarter flow recorded at the terminal aorta increases in the transposition response, induced by transposing the rat to a new cage (IRIUCHIJMA et al., 1982). On the other hand, during grooming of the rat, hindquarter flow decreases when carotid flow increases (Mizuma et al., 1987). In both occasions, arterial pressure remains almost unchanged or slightly increases despite the profuse increase in blood flow in some regions. It seems that excitation of sympathetic vasoconstrictor fibers supplying resistance vessels of the areas for compensatory vasoconstriction is coupled with the act which increases regional flow in a feed-forward "fixed action pattern" triggered by a suitable central command (SHEPHERD, 1983).

MillHORN et al. (1987) have provided experimental support for the central command hypothesis for the genesis of the respiratory hyperpnea and increased cardiovascular function that occur during exercise in the cat. This report stimulated us to study those areas where compensatory vasoconstriction might occur in the spontaneously walking rat when one would expect a marked increase in hindquarter flow. In the present study, regional blood flow was observed with implanted electromagnetic flow probes during spontaneous walk of this animal in a wheel.

\section{METHODS}

Implantation of flow probe and catheters. Male Wistar rats 10-20 weeks old and weighing $250-450 \mathrm{~g}$ were anesthetized with thiamylal sodium $(50 \mathrm{mg} / \mathrm{kg}$, i.p. $)$. An electromagnetic flow probe (Nihon Kohden type FC with internal diameter of 1-2 mm) was implanted around the right carotid, superior mesenteric, or left renal artery, or the terminal aorta. The technical details are described elsewhere (KAWAUE and IRIUCHIJIMA, 1984). The lead wire of the flow probe ending in a plug was passed under the skin and led to the dorsal neck. There the outer end of the wire was sutured to neck muscles so that only the plug was exposed on the skin. A polyethylene catheter (PE10 fused to PE20) for arterial pressure measurement was inserted into the terminal aorta via the right femoral artery or into the aortic arch via the right common carotid artery. Another catheter was inserted into the right external jugular vein for intravenous injection. The distal end of each catheter, which was plugged with a short stainless steel wire stopper when not in use, was also led to the dorsal neck as the lead wire from the flow probe.

Observations of flow and pressure. After implantation, the rat was kept separately in a $35 \times 30 \times 17 \mathrm{~cm}$ white polyethylene cage containing wood chips. Water and pellets were given ad libitum. Flow and pressure measurements were started 3 days after implantation when the rat had resumed taking ample amounts of water and pellets. With the rat in its home cage, and after removal of the metal wire top of the cage which contained pellets and a water bottle in its indentation, an external cable from the flowmeter circuit (Nihon Kohden, MFV-1100) was connected to the plug of the flow probe. A polyethylene tube from a pressure transducer was connected to the arterial catheter after the stopper was removed. 
Flow and pressure signals were smoothed with RC circuits with a time constant of $1 \mathrm{~s}$ to facilitate the reading of mean values. Each flow probe was calibrated before implantation by passing known amounts of $0.9 \%$ saline through an excised artery. Flow was expressed in units of $\mathrm{ml} /(\mathrm{min} \cdot 100 \mathrm{~g}$ body weight) and pressure in $\mathrm{mmHg}$. Flow and pressure were recorded with a pen writing oscillograph.

Wheel walk. The wheel into which the rat was transposed from the home cage by the experimenter's hand for walking was in the shape of a flat cylinder with a diameter of the base of $35 \mathrm{~cm}$ and a height of $10 \mathrm{~cm}$. One base was made of stainless steel and the other was open. At the center of the outside of the metal base a perpendicular pivot was fitted, which was kept horizontally and could rotate smoothly in a ball bearing. The side of the cylinder was made of wire mesh, on the internal surface of which the rat was placed so that he could walk at will. The wheel rotated as the rat walked, leaving it at almost the same place in space. After placing the rat inside the wheel, one side of an empty white polyethylene cage was placed close to the open base of the cylinder lest the rat should come out. Walking of the rat was marked manually on recording paper. Sometimes the walking speed was calculated from the time required for a known angle of rotation of the wheel, measured with a stop watch. Rats which often would walk spontaneously in the wheel were selected for the present study. After transposing the rat into the wheel, we waited for about $10 \mathrm{~min}$ before starting observations of flow and pressure so that these variables became stationary when the rat was motionless.

Observations of heart rate. In some rats heart rate was measured by a special heart rate meter. In most of them only the arterial and venous catheters had been implanted. The arterial pressure signal was amplified and fed to a Schmidt trigger producing one pulse for each heart beat. The triggering output was led to a $50 \mathrm{~ms}$ square pulse generator, the output of which was smoothed with an RC circuit with a time constant of $1 \mathrm{~s}$ before entering a channel of the pen writing oscillograph. Thus successive change of heart rate was presented as vertical deflection of the pen. This heart rate meter was calibrated by feeding a train of pulses at frequencies of 300,400 , and $500 / \mathrm{min}$ to the square pulse generator. Since the smoothing with the $\mathrm{RC}$ circuit was incomplete, at slower speeds of the recording paper, the recording of heart rate was made as a band instead of a line (Figs. 2 and 5). The center of the band was read as the instantaneous heart rate in beats $/ \mathrm{min}$.

Cervical sympathectomy. Some rats with a flow probe at the carotid artery were anesthetized briefly by intravenous injection of thiamylal sodium at a dose of $25 \mathrm{mg} / \mathrm{kg}$. The right cervical sympathetic nerve was cut immediately caudally to the superior cervical ganglion. Measurements of flow and pressure were resumed two days later.

RESULTS

Hindquarter flow

Figure 1 gives one example of recording of arterial pressure and hindquarter 


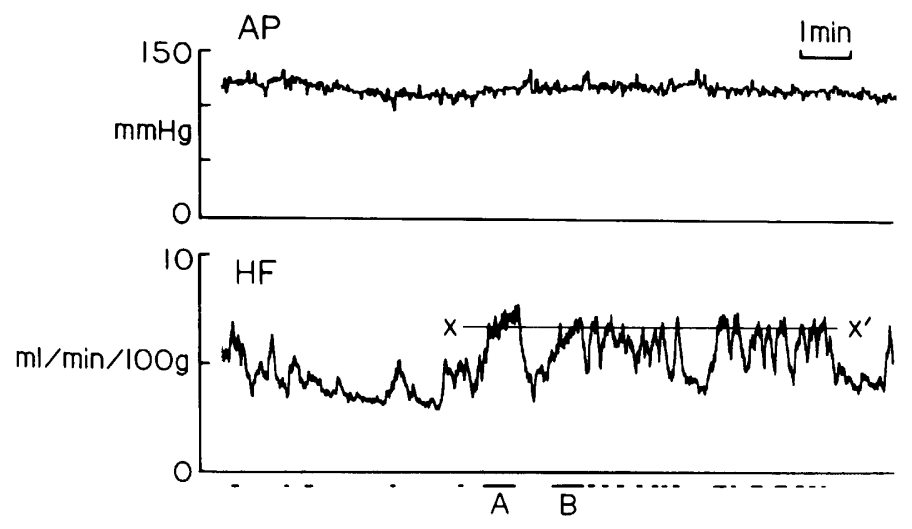

Fig. 1. Simultaneous recording of arterial pressure (AP) and hindquarter flow (HF) measured at the terminal aorta in a rat. Wheel walk for the underlined periods. For the walks marked A and B, the walk speed was both about $14 \mathrm{~cm} / \mathrm{s}$. The horizontal line $\mathrm{X}-\mathrm{X}^{\prime}$ was drawn to approximate the average level during walking.

flow in a rat placed in the wheel. The rat walked spontaneously for the underlined periods. The speed of walk for the stretches marked A and B was calculated for both as about $14 \mathrm{~cm} / \mathrm{s}$. The method of measurement of the speed was applicable only for such walks of longer duration. The speed as well as the duration of walks varied widely. The level which blood flow reached while walking was also variable for each walk but, as a crude approximation, the horizontal line $\mathrm{X}-\mathrm{X}^{\prime}$ was drawn in Fig. 1 as the average level during walking, which was $6.7 \mathrm{ml} /(\mathrm{min} \cdot 100 \mathrm{~g})$ for this particular example. The resting level of hindquarter flow was similarly determined as $4.1 \mathrm{ml} /(\mathrm{min} \cdot 100 \mathrm{~g})$ for this rat. This kind of treatment was only semi-quantitative but was considered to be of some value in obtaining a rough idea about the redistribution of cardiac output during walking. The mean blood flow was given to only two significant figures because of its semi-quantitative nature. Carotid flow and heart rate were treated in a similar fashion. Arterial pressure did not change in relation to walks. The mean levels of hindquarter flow at rest and during walking from 7 rats were $3.8 \pm 1.1$ and $6.7 \pm 1.7 \mathrm{ml} /(\mathrm{min} \cdot 100 \mathrm{~g})$, respectively. The difference is significant at $p<0.001$ by the paired $t$-test.

\section{Carotid flow}

Carotid flow decreased whenever the rat walked in the wheel. An example is presented in Fig. 2, left. The mean carotid flow \pm S.D. from 5 rats at rest was $1.2 \pm 0.2 \mathrm{ml} /(\mathrm{min} \cdot 100 \mathrm{~g})$ and during walking was $0.9 \pm 0.2$. The difference is significant at $p<0.001$. The decrease in carotid flow during wheel walking disappeared after severance of the cervical sympathetic nerve (Fig. 2, right).

\section{Superior mesenteric flow}

Blood flow in this region was observed in 6 rats. In three the blood flow did 
AFTER CERV. SYMPATHECT.
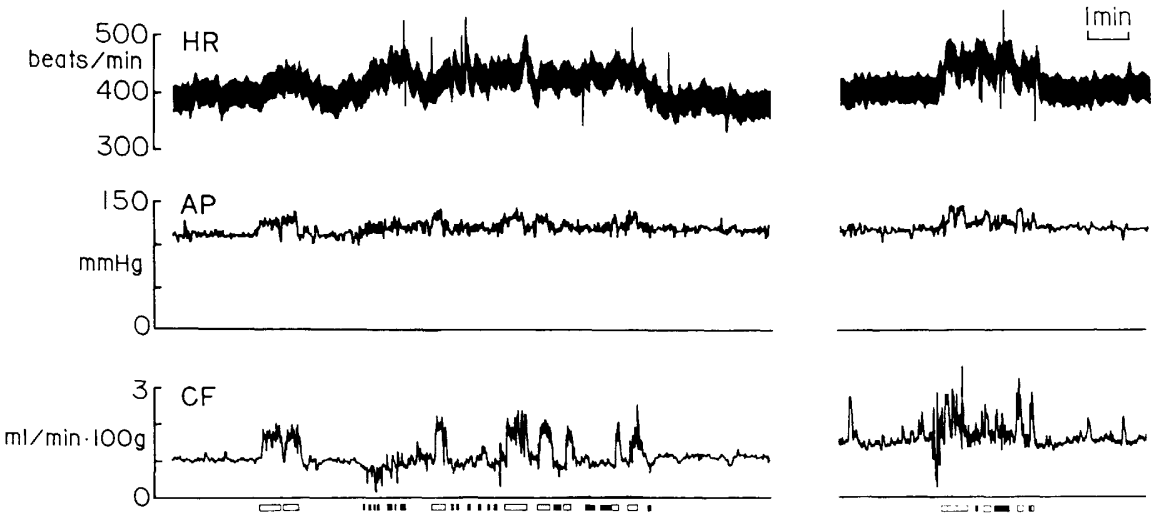

Fig. 2. From top to bottom, for both left and right parts, heart rate (HR), arterial pressure (AP), and carotid flow (CF). For the periods represented by closed rectangles at the bottom, the rat was walking; for open rectangles, grooming. Left: before cervical sympathectomy; right: 2 days after sympathectomy. During walking, carotid flow decreased before sympathectomy but did not after sympathectomy.

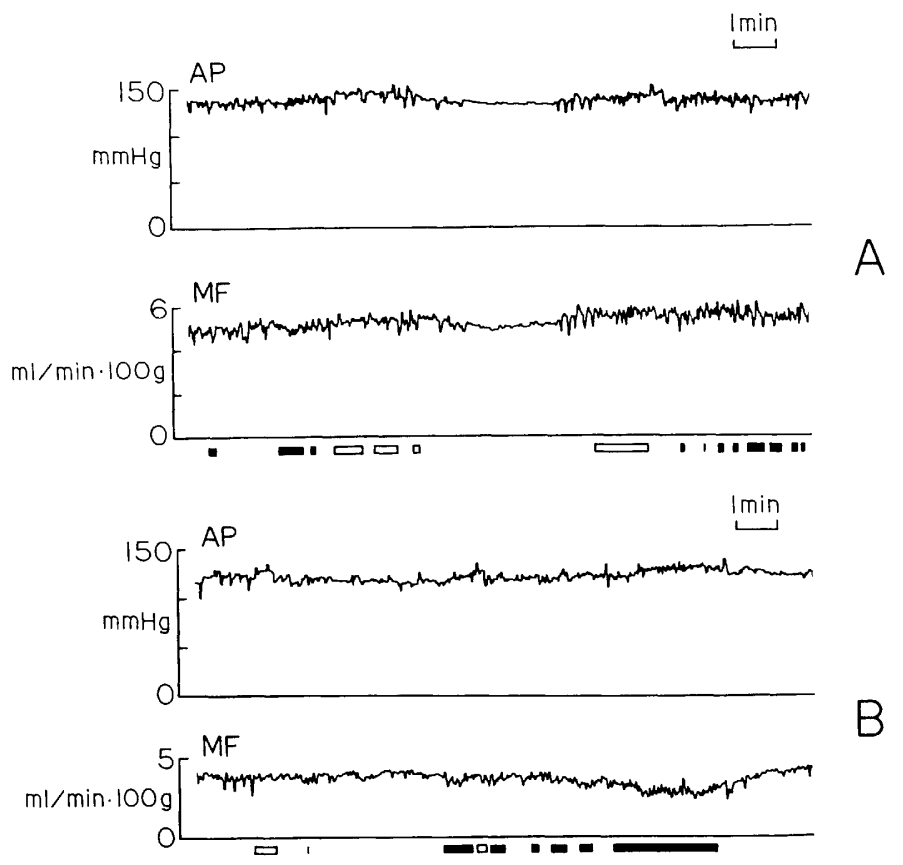

Fig. 3. Simultaneous recording of arterial pressure (AP) and superior mesenteric flow (MF) in two different rats. Symbols for walking and grooming as in Fig. 2. In A, superior mesenteric flow remained almost unchanged during walking. In $\mathrm{B}$, the flow decreased. 

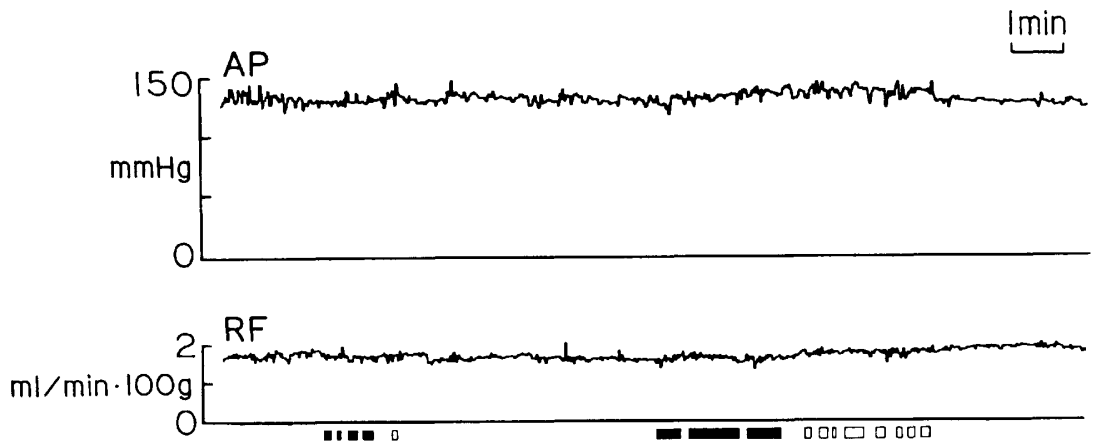

Fig. 4. Simultaneous recording of arterial pressure (AP) and renal flow (RF) in a rat. Symbols for walking and grooming as in Fig. 2. Neither walking nor grooming induced appreciable changes in renal flow.

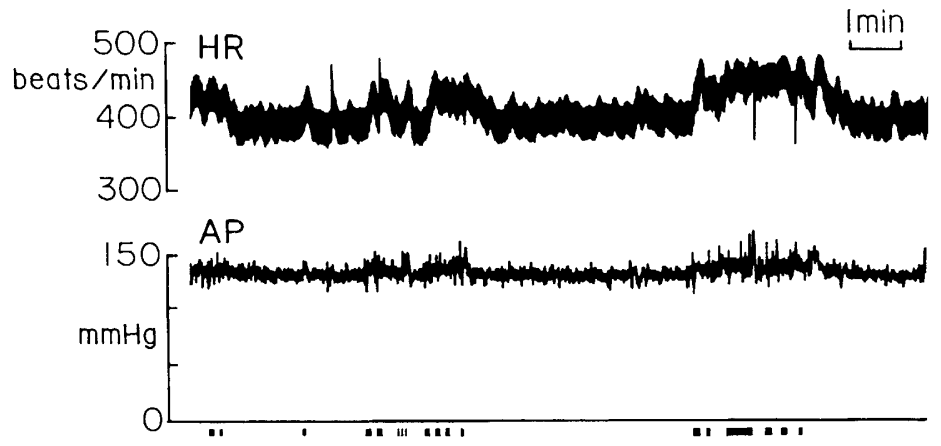

Fig. 5. Simultaneous recording of heart rate (HR) and arterial pressure (AP) in a rat placed in the wheel. The rat walked for the underlined periods.

not appear to change in causal relation to walking (Fig. 3A), while in the three others the flow tended to decrease during wheel walking, especially for long time durations (Fig. 3B). The mean values for this and renal flows at rest are to be found in our previous paper (IRIUCHIJIMA et al., 1982).

\section{Renal flow}

Renal flow was observed in 4 rats. No flow changes attributable to wheel walk were observed in any of the rats (Fig. 4).

\section{Heart rate}

An example of simultaneous recording of heart rate and arterial pressure in a rat in the wheel is presented in Fig. 5. Heart rate increased about $10 \%$ whenever the rat walked in the wheel: the mean heart rate \pm S.D. from 7 rats was $397 \pm 42$ beats/min at rest and $449 \pm 42$ during walking. The difference is significant at $p<0.005$. 


\section{DISCUSSION}

During spontaneous wheel walk of the rat, when the hindquarter flow increased, the common carotid flow decreased, through excitation of cervical sympathetic vasoconstrictor fibers. This regional sympathetic activity seems to be in some way coupled with the act of walking in a fixed action pattern. This reciprocity between hindquarter and carotid flows during walking is interesting in connection with another reciprocity: that between the same two flows in the inverse direction during grooming (Mizuma et al., 1987). The spinal segments supplying vasoconstrictor outflow to the regions of these two flows are widely separated. This anatomical arrangement may be related with the above reciprocity in either direction. The behavior of flows in the regions supplied by the segments in between was alike for both walking and grooming: Superior mesenteric flow decreased in some rats and renal flow remained unchanged in all rats.

As in the study of grooming (Mizuma et al., 1987) this study also depended on the behavioral caprice of the rat. Although rats which frequently walked in the wheel were selected before implantation, some rats would not walk after implantation and had to be discarded. Even for those which walked in the wheel spontaneously after implantation, during observations of flow and other measurements, frequency, duration and speed of walking were variable even in the same rat. Therefore, statistical results given in this paper are inevitably only semi-quantitative. Nevertheless, we may safely estimate that the average decrease in common carotid flow during walking of about $0.6 \mathrm{ml} /(\mathrm{min} \cdot 100 \mathrm{~g})$ body weight, counting the bilateral carotids, was far too low to compensate for the increase of hindquarter flow which averaged about $3 \mathrm{ml} /(\mathrm{min} \cdot 100 \mathrm{~g})$.

Despite this deficit the arterial pressure did not decrease. Since the decrease in superior mesenteric flow was inconstant and, even when it occurred, it was too small to compensate for the increase in hindquarter flow. Substantial flow decreases in areas other than those to which flow probes were applied seem unlikely. Here we are forced to assume an immediate increase in cardiac output. We say "immediate," because cardiac output would eventually increase after recirculation of the increased hindquarter flow. With the increase in cardiac output by recirculation alone, however, arterial pressure should have decreased at the beginning of a wheel walk. The observed immediate increase in heart rate of about $10 \%$ on the average would be enough to compensate for the increase in hindquarter flow, if minute volume increased proportionately to heart rate with suitable cooperative constriction of the capacitance sections. Cardiac output of the rat at rest is about $20 \mathrm{ml} /(\mathrm{min} \cdot 100 \mathrm{~g}$ body weight) (IRIUCHIJIMA et al., 1980). Ten percent of this or about $2 \mathrm{ml} /(\mathrm{min} \cdot 100 \mathrm{~g})$ is similar in magnitude to the increase in hindquarter flow.

The above reasoning indicates that the observed decrease in carotid flow during the rat's wheel walk is quantitatively unimportant in impeding a drop in arterial pressure. This mechanism might have been of more value at a certain stage of 
evolution. However, the observed result gives a clue to how finely regional sympathetic nerves are controlled in conscious animals. It should be noted that wheel walk was not accompanied by a marked decrease in visceral blood flow: The decrease in superior mesenteric flow was inconstant and renal flow remained unchanged. Decrease in visceral flow or increase in visceral flow resistances with elevated arterial pressure during exercise have been reported in dogs (RUSHMER et al., 1961; VATNER et al., 1971), in cats (MANCIA et al., 1974; MARSHALl and TimMS, 1980; Diepstra et al., 1982), and in baboons (VATNER, 1978; HoHIMER et al., 1983). Presumably, voluntary walk observed in the rat in this study is usually not so stressful as to induce visceral vasoconstriction and arterial pressure elevation. The change of the visceral flow during wheel walk is comparable with that during grooming (Mizuma et al., 1987). Perhaps for rats these two behaviors are equally comfortable. It is evident that the mesenteric and renal beds are amply innervated, because they respond with marked vasoconstriction when the animal is held by the experimenter's hand and is transposed to a new cage (IRIUCHIJIMA et al., 1982). Stability of mesenteric and renal flows during violent, prolonged exercise has been observed in Alaska sled dogs by VAN CiTTERS and Franklin (1969). They consider that redistribution of blood flow away from the kidney and viscera might be inappropriate or even disastrous.

In summary, the area where vasoconstriction took place most consistently during the rat's spontaneous wheel walk when hindquarter flow increased was that supplied by the common carotid artery. The vasoconstriction was mediated by the cervical sympathetic nerve. However, quantitatively, it was insufficient to compensate for the increased blood drain in the hindquarter areas. The more important compensatory mechanism for maintaining arterial pressure seemed to be an increase in cardiac output which was suggested by the observed increase in heart rate.

\section{REFERENCES}

Diepstra, G., Gonyea, W., and Mitchell, J. H. (1982) Distribution of cardiac output during static exercise in the conscious cat. J. Appl. Physiol., 52: 642-646.

Franklin, D. L., Watson, N. W., and Van CitTers, R. L. (1964) Blood velocity telemetered from untethered animals. Nature, 203: 528-530.

Hohimer, A. R., Hales, J. R., Rowell, L. B., and Smith, O. A. (1983) Regional distribution of blood flow during mild dynamic leg exercise in the baboon. J. Appl. Physiol., 55: 1173-1177.

Iriuchijima, J., KawaUe, Y., and Teranishi, Y. (1982) Blood flow redistribution in the transposition response of the rat. Jpn. J. Physiol., 32: 807-816.

Iriuchijima, J., Teranishi, Y., and Eguchi, C. (1980) Transposition response, a cardiovascular response to change of habitat in the rat. Jpn. J. Physiol., 30: 887-896.

Kawaue, Y. and IrIUChiJima, J. (1984) Changes in cardiac output and peripheral flows on pentobarbital anesthesia in the rat. Jpn. J. Physiol., 34: 283-294.

MANCIA, G., BACCELlI, G., and ZANCHETTI, A. (1974) Regulation of renal circulation during 
behavioral changes in the cat. Am. J. Physiol., 227: 536-542.

Marshall, J. M. and Timms, R. J. (1980) Experiments on the role of the subthalamus in the generation of the cardiovascular changes during locomotion in the cat. J. Physiol. (Lond.), 301: 92P-93P.

Millhorn, D. E., Eldridge, F. L., Waldrop, T. G., and Kiley, J. P. (1987) Diencephalic regulation of respiration and arterial pressure during actual and fictive locomotion in cat. Circ. Res., 61: I-53-I-59.

Mizuma, E., Takemoto, Y., and Iriuchijima, J. (1987) Redistribution of cardiac output during grooming of the rat. Jpn. J. Physiol., 37: 49-57.

Rushmer, R. F., Franklin, D. L., Van Citters, R. L., and Smith, O. A. (1961) Changes in peripheral blood flow distribution in healthy dogs. Circ. Res., 9: 675-687.

SHEPHERD, G. M. (1983) Neurobiology, Oxford Univ. Press, Oxford, p. 364.

VAN Citters, R. L. and Franklin, D. L. (1969) Cardiovascular performance of Alaska sled dogs during exercise. Circ. Res., 24: 33-42.

VATNER, S. F. (1978) Effects of exercise and excitement on mesenteric and renal dynamics in conscious, unrestrained baboons. Am. J. Physiol., 232: $\mathrm{H} 210-\mathrm{H} 214$.

Vatner, S. F., Higgins, C. B., White, S., Patrick, T., and Franklin, D. (1971) The peripheral vascular response to severe exercise in untethered dogs before and after complete heart block. J. Clin. Invest., 50: 1950-1960. 